\title{
CURVAS DE CRESCIMENTO E DE ÍNDICE DE LOCAL PARA POVOAMENTOS DE Tectona grandis EM TANGARÁ DA SERRA, MATO GROSSO ${ }^{1}$
}

Jovane Pereira da Cruz ${ }^{2}$, Helio Garcia Leite ${ }^{3}$, Carlos Pedro Boechat Soares ${ }^{3}$, João Carlos Chagas Campos ${ }^{3}$, Luit Smit ${ }^{4}$ Gilciano Saraiva Nogueira ${ }^{4}$

\begin{abstract}
RESUMO - Este estudo teve por objetivo avaliar as tendências de crescimento de variáveis do povoamento e avaliar alternativas para construção de curvas de índice de local para teca (Tectona grandis), em plantios localizados na região de Tangará da Serra - Mato Grosso. Para isso, foram utilizados dados de 50 parcelas permanentes com no mínimo quatro medições sucessivas. As tendências de crescimento em altura, diâmetro, área basal e volume foram analisadas por meio da análise de regressão, utilizando o modelo Chapman-Richards. Dois métodos de construção de curvas de índices de local, com diferentes modelos, foram avaliados. Após análises, verificou-se que o modelo Chapman-Richards ajustou-se bem aos dados observados, descrevendo as tendências de crescimento das variáveis dos povoamentos. A melhor alternativa para construir curvas de índices de local foi o método da curva-guia, empregando o modelo Chapman-Richards.
\end{abstract}

Palavras-chave: Tectona grandis, índice de local, modelo de Chapman-Richards.

\section{GROWTH AND SITE INDEX CURVES FOR Tectona grandis STANDS IN TANGARÁ DA SERRA, MATOGROSSO STATE, BRAZIL}

\begin{abstract}
The objective of this study was to evaluate the growth tendencies of the stand variables and evaluate alternatives to construct site index curves for teak (Tectona grandis) in stands located in the Tangará da Serra, Mato Grosso state. For this purpose, data from 50 permanent plots with a minimum of four successive measurements were used. The tendencies of growth in height, diameter, basal area and volume were analyzed by means of the regression method, using the Chapman-Richards model. Two methods of construction of site index curves, with different models, were evaluated. The results of the analysis showed that ChapmanRichards model adjusted well to the observed data, describing the growth tendencies of the stand variables. The best alternative for the construction of site index curves was the guide-curve method, using the ChapmanRichards model.
\end{abstract}

Key words: Tectona grandis, site index, Chapman-Richards model.

\footnotetext{
${ }^{1}$ Recebido em 06.10.2006 e aceito para publicação em 19.05.2008.

${ }^{2}$ Programa de Pós-Graduação em Ciência Florestal da Universidade Federal de Viçosa (UFV), Viçosa-MG.

${ }^{3}$ Departamento de Engenharia Florestal da UFV. E-mail: <hgleite@gmail.com>.

${ }^{4}$ Faculdade de Ciências Agrárias da Universidade Federal dos Vales do Jequitinhonha e Mucuri. E-mail: <nogueirags@yahoo.com.br>
} 


\section{INTRODUÇÃO}

Muitos estudos em mensuração têm sido conduzidos no Brasil utilizando dados de povoamentos do gênero Eucalyptus e Pinus. Entretanto, estudos desta natureza são escassos para teca (Tectona grandis), espécie de origem asiática, muito valorizada no mercado internacional pela qualidade de sua madeira, e que está sendo cultivada em grande escala no Mato Grosso.

Dentro do contexto da mensuração florestal no Brasil, destaca-se o trabalho de Macedo et al. (1999), que avaliou o crescimento inicial em plantios de teca em Minas Gerais, e o trabalho de Nogueira (2003), que avaliou crescimento e produção de povoamentos de teca submetidos a desbaste através de modelos de distribuição diamétrica. Em relação a outros países, Keogh (1990) e Haishui (1993), estudaram os padrões de crescimento de teca no Caribe e América Central e nas Ilhas Hainan, respectivamente.

Embora existam estudos sobre a tendência de crescimento de árvores e povoamentos de teca, muitos deles se baseiam em funções empíricas, sem significado biológico. De acordo com Campos e Leite (2002) estudos desta natureza devem se basear em modelos que possuam pressuposições biológicas, como por exemplo, o modelo de Chapman-Richards (Pienaar e Turnbull, 1973; Zeide, 1993).

Tendo em vista a carência de informações acerca da biometria e do crescimento inicial de teca no Brasil, este estudo foi conduzido com o objetivo de avaliar as tendências de crescimento de variáveis do povoamento, e também avaliar alternativas para construção de curvas de índice de local para teca na região de Tangará da Serra, Mato Grosso.

\section{MATERIAL E MÉTODOS}

Para este estudo, foram utilizados dados de inventário contínuo, realizados em plantios de teca, com espaçamento inicial entre plantas de 3,0 x 3,0 m, na região de Tangará da Serra, Estado do Mato Grosso, Brasil. Um total de 50 parcelas, com área de 765 m², medidas aos 22, 34, 46 e 58 meses foram utilizadas para as análises. Dentro de cada parcela, foram medidos os diâmetros com casca a 1,30 m do solo (dap) e as alturas totais $(\mathrm{Ht})$ de todas as árvores.

Visando o ajuste de modelos volumétricos, 60 árvores-amostra representativas das classes de diâmetro observadas nas quatro idades foram abatidas e cubadas pela aplicação sucessiva da expressão de Smalian, em seções de 1,0 m de comprimento.

Foram ajustadas equações para fornecer as estimativas de volume com e sem casca para as árvores individuais, tendo como base o modelo de Schumacher e Hall (1933), assim definido:

$$
\operatorname{Ln}(V)=\beta_{0}+\beta_{1} \operatorname{Ln}(\text { dap })+\beta_{2} \operatorname{Ln}(H t)+\varepsilon
$$

em que

$V=$ volume, com ou sem casca, em $\mathrm{m}^{3}$;

dap = diâmetro com casca a 1,30 m, em cm;

$H t=$ altura total, em m;

$L n=$ logaritmo neperiano;

$\beta_{0} \ldots \beta_{2}=$ parâmetros do modelo; e

$\varepsilon=$ erro aleatório, $\sim \operatorname{NID}\left(0, \sigma^{2}\right)$.

As equações de volume ajustadas foram avaliadas pelos seguintes critérios: a) análise gráfica dos resíduos ou erros, em percentagem (E\%), obtidos por:

$$
E \%=\frac{\hat{\text { Vestimado }- \text { Vobservado }}}{\text { Vobservado }} .100 \text {, }
$$

b) gráficos de dispersão dos volumes estimados em relação aos volumes observados; c) gráficos do volume de casca e da percentagem de casca em relação ao dap; e d) coeficiente de determinação $\left(\mathrm{R}^{2}\right)$ e coeficiente de variação (CV) (Campos e Leite, 2002).

A equação de volume com casca, para árvores individuais, foi utilizada para estimar o volume com casca por hectare $(V c c)$ das parcelas. Além das estimativas de volume por unidade de área, obtiveramse, também, as estimativas de diâmetro médio (q), de área basal por hectare $(B)$ e de altura dominante (média das alturas totais de cinco árvores dominantes por parcela) e altura total média (Ht ) por parcela.

Para caracterizar as tendências de crescimento das variáveis diâmetro médio (q), área basal por hectare $(B)$, altura dominante $(H d)$ e altura total média $(H t)$ e volume com casca por hectare $(V c c)$, foi ajustado o modelo de Chapman-Richards, assim definido:

$$
y=\beta_{0}\left(1-e^{-\beta_{1} I}\right)^{\beta_{2}} . \varepsilon
$$

em que

$$
\begin{aligned}
& y=\text { variável analisada }(q, H t, H d, B \text { e } V c c) ; \\
& I=\text { idade, em meses; }
\end{aligned}
$$


$\beta_{0}=$ assíntota; $\beta_{1}=$ ponto de inflexão; $\beta_{2}=$ taxa de crescimento.

$\varepsilon=$ erro aleatório, $\sim \operatorname{NID}\left(0, \sigma^{2}\right)$.

Para construir curvas de índice de local foram avaliadas as seguintes alternativas:

1) método da curva-guia e o modelo ChapmanRichards:

$$
H d=\beta_{0}\left(1-e^{-\beta_{1} I}\right)^{\beta_{2}} . \varepsilon ;
$$

2) método de atribuição preliminar de índice de local e o modelo proposto por Payandeh e Wang (1995):

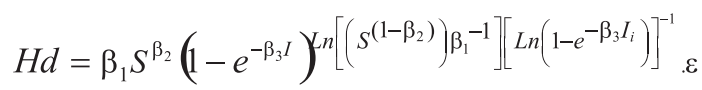

em que

$$
\begin{aligned}
& H d=\text { altura dominante, em metros; } \\
& S=\text { índice de local; } \\
& I=\text { idade, em meses; } \\
& I_{i}=\text { idade índice; em meses; } \\
& \beta_{0}, \beta_{1}, \beta_{2}, \beta_{3}=\text { parâmetros dos modelos. } \\
& \varepsilon=\text { erro aleatório, } \sim \operatorname{NID}\left(0, \sigma^{2}\right) .
\end{aligned}
$$

Para empregar a alternativa 2, inicialmente foram estimados os índices de local preliminares por meio do seguinte modelo de regressão:, ajustado para cada parcela, conforme sugerido por Campos e Leite (2002).

\section{RESULTADOS E DISCUSSÃO}

\subsection{Equações de volume}

As equações de volume com casca $(V c / c)$ e sem casca $(V s / c)$ por árvore, e respectivas estatísticas de precisão, foram:

$\operatorname{Ln}\left(V_{C} / c\right)=-8,57046+1,07588 \operatorname{Ln}($ dap $)+$ $1,27429 \operatorname{Ln}(H t), R^{2}=0,987$ e $C V=3,54 \%$; e $\operatorname{Ln}\left(V_{c} / c\right)=$ $-9,26092+1,12832 \operatorname{Ln}($ dap $)+1,36535 \operatorname{Ln}(H t), R^{2}=0,983$ e $C V=3,77 \%$.

A análise dos gráficos na Figura 1 e as estimativas dos coeficientes de determinação $\left(R^{2}\right)$ e dos coeficientes de variação $(C V)$ permitem afirmar que essas equações são precisas e não-tendenciosas, podendo ser utilizadas em inventários de povoamentos de teca conduzidos em idades inferiores a 6 anos.

\subsection{Tendências de crescimento das características do povoamento}

As variáveis altura dominante $(H d)$, diâmetro médio $(q)$, área basal por hectare $(B)$ e volume com casca por hectare $(V c c)$ tiveram uma tendência sigmóide bem definida, conforme apresentado na Figura 2, indicando a coerência da utilização do modelo de Chapman-Richards para descrever o comportamento das variáveis.

As estimativas dos parâmetros referentes ao ajustes do modelo de Chapman-Richards para as variáveis diâmetro médio $(q)$, área basal $(B)$, altura dominante $(H d)$ e volume com casca $(V c c)$, encontram-se no Quadro 1.

Todas as estimativas dos parâmetros foram significativas em nível de $95 \%$ de probabilidade $(p \leq 0,05)$ pelo teste t. Além disso, correlações acima de 87\% entre os valores observados e estimados para as variáveis avaliadas indicam que o modelo de Chapman-Richards foi eficiente para descrever as tendências de crescimento das características do povoamento analisado.

Visando permitir a elaboração de tabelas de volume de dupla entrada para uso em inventários florestais, foi gerada a relação hipsométrica: $H t=20,2143\left(1-e^{-0,09625 d a p}\right)^{1,5733}$, com $r_{y \hat{y}}=0,894$. Esta equação foi obtida utilizando os dados de todas as idades. Como complemento, foram ajustadas equações agrupando-se os dados por classe de idade para verificar possíveis efeitos da idade na relação hiposmétrica. Com base nas equações apresentadas na Figura 3, verifica-se a necessidade do uso de equações independentes, haja vista a grande diferença entre os coeficientes das equações, indicando comportamentos distintos para as curvas de altura. Assim sendo, recomenda-se utilizar relações hipsométricas específicas para cada ano do inventário florestal contínuo.

O realismo biológico e qualidade do ajuste permitem inferir que o modelo de Chapman-Richards é adequado para descrever o crescimento em altura de teca. De fato, Piennar e Turnbull (1973) já haviam constatado que este modelo é capaz de descrever adequadamente o crescimento individual de árvores.

\subsection{Curvas de Índice de Local}

Para a alternativa 1, que utiliza o método da curvaguia e o modelo Chapman-Richards, as curvas de índice de local foram geradas a partir da equação:

R. Árvore, Viçosa-MG, v.32, n.4, p.679-685, 2008 


$$
H d=S\left[\frac{\left(1-e^{-0,0252 I}\right)^{1,3221}}{\left(1-e^{-0,0252 I_{i}}\right)^{1,3221}}\right], \quad I_{i}=48 \text { meses. }
$$

Os coeficientes são aqueles apresentados no Quadro 1 para a variável altura dominante $(H d)$. A idade-
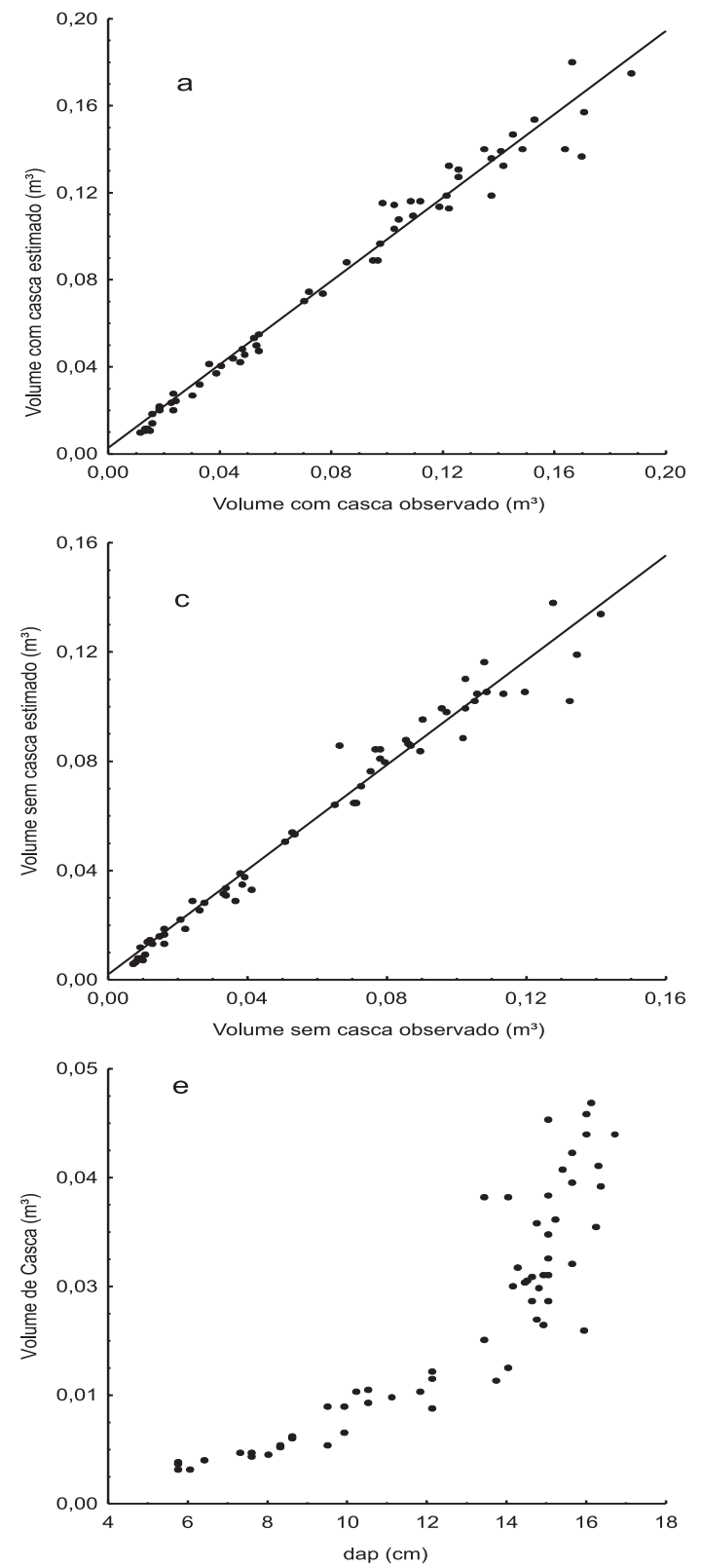

índice $\left(I_{i}\right)$ utilizada foi de 48 meses.

Na Figura 4 são apresentas curvas de índice de local geradas a partir da equação anterior. As Classes I, II e III referem-se a locais "bons”, “médios” e "ruins”, respectivamente, em termos de capacidade produtiva.
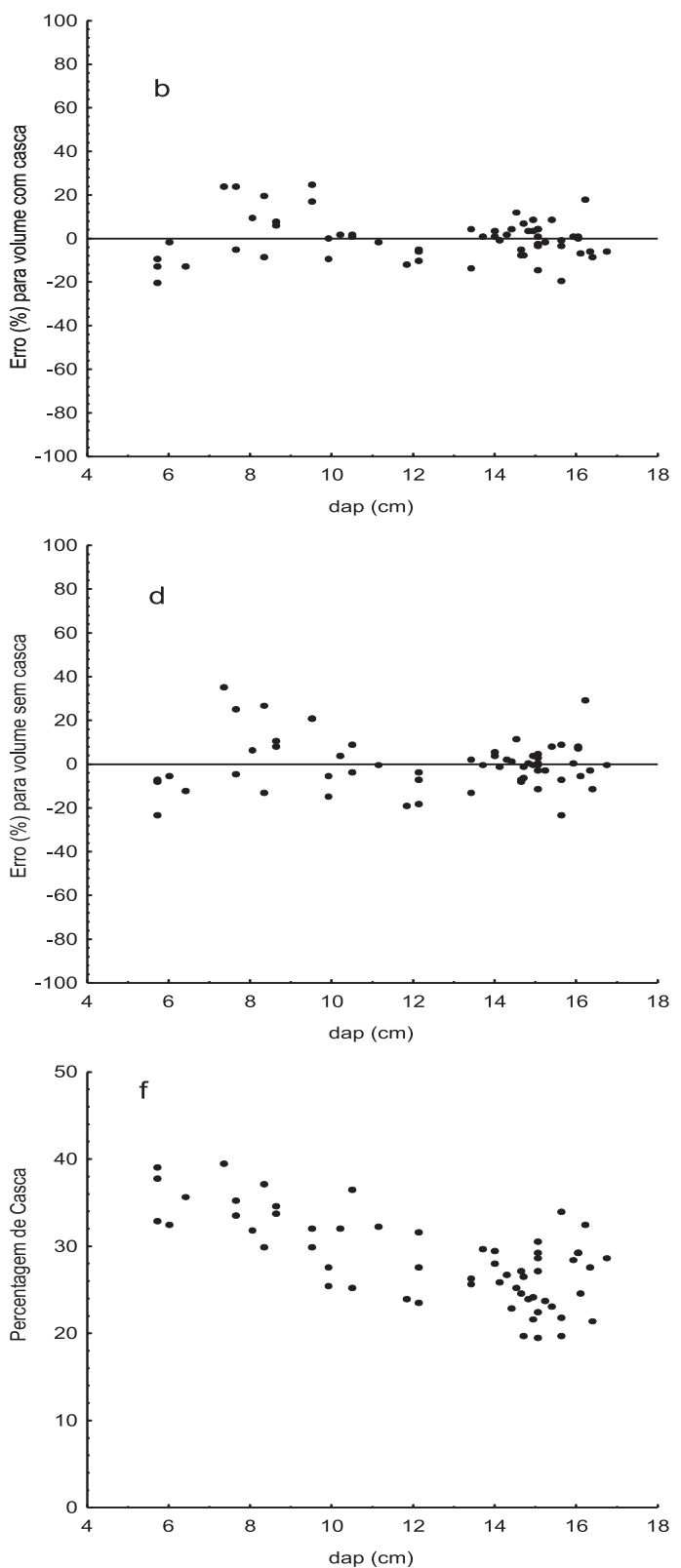

Figura 1 - Volumes estimados em relação aos volumes observados ( $a$ e $c$ ); e erros percentuais em relação ao dap $(b$ e $d$ ); volume de casca e percentagem de casca em relação ao dap ( $e$ e $f$ )

Figure 1 - Estimated volumes in relation to observed volumes ( $a$ and c); percentage of errors related to d.b.h ( $b$ and d); volume and percentage of bark related to d.b.h ( $e$ and $f$ ).

R. Árvore, Viçosa-MG, v.32, n.4, p.679-685, 2008 

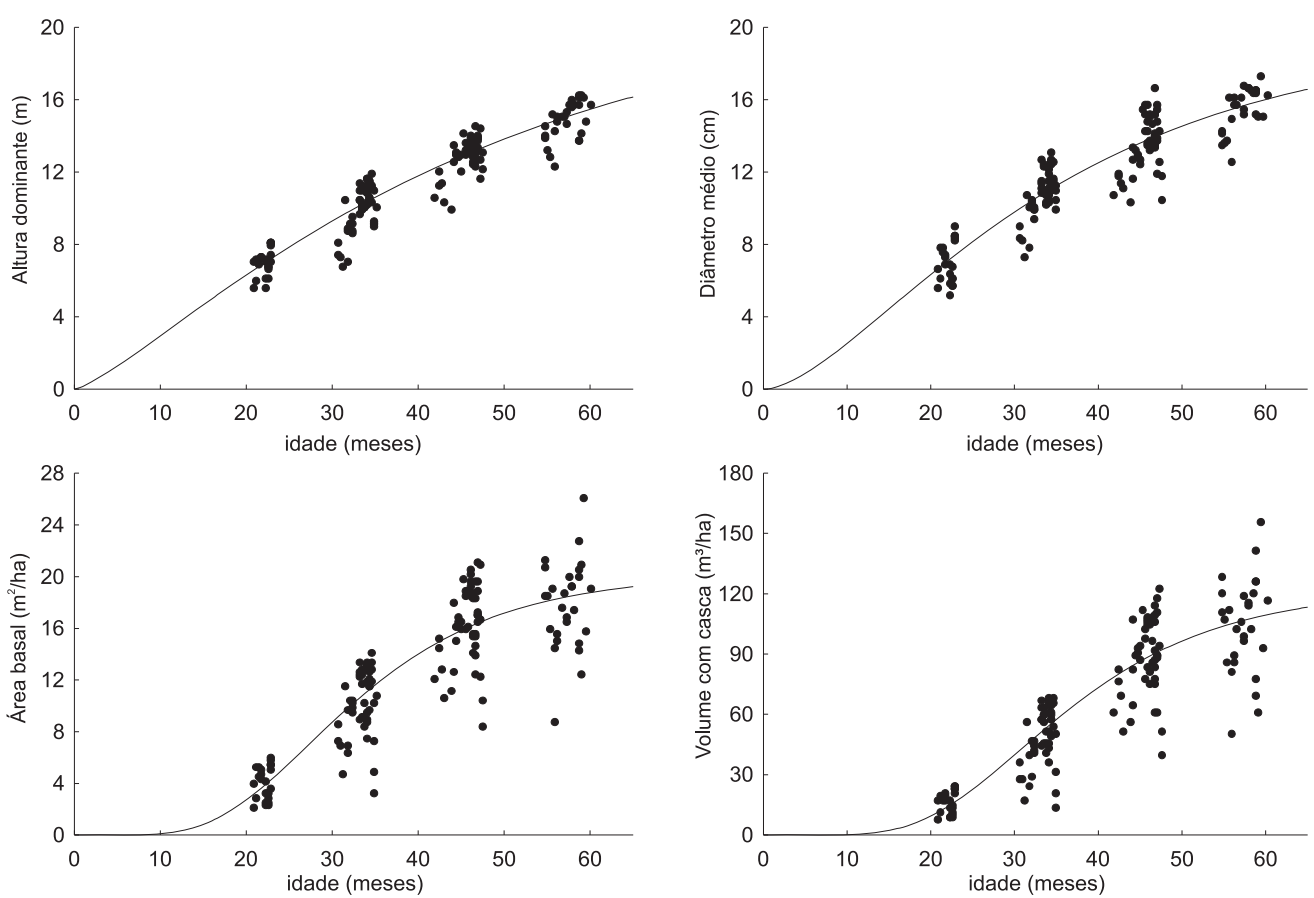

Figura 2 - Tendências de crescimento das variáveis altura dominante (a), diâmetro médio (b), área basal por hectares (c), e volume com casca por hectare $(d)$.

Figure 2 - Tendencies of growth in dominat height (a), quadratic diameter (b), basal area per hectare (c) and volume outside bark per hectare $(d)$.

Quadro 1 - Estimativas dos parâmetros do modelo de Chapman-Richards para as variáveis diâmetro médio ( $q$ ), área basal por hectare $(B)$, altura dominante $(H d)$ e volume com casca $(V C c)$, com os respectivos coeficientes de correlação $\left(r_{y \hat{y}}\right)$

Table 1 - Estimates of the Chapman-Richards model parameters for quadratic diameter (q), basal area per hectare (B), dominant height $(\mathrm{Hd})$ and volume outside bark $(\mathrm{VcC})$, with respective correlation coefficients $\left(r_{y \hat{y}}\right)$

\begin{tabular}{ccccc}
\hline Estimativas dos Parâmetro & $q(\mathrm{~cm})$ & $B\left(\mathrm{~m}^{2} / \mathrm{ha}\right)$ & $H d(\mathrm{~m})$ & $V c c\left(\mathrm{~m}^{3} / \mathrm{ha}\right)$ \\
\hline$\hat{\beta_{0}}$ & 19,4183 & 20,1370 & 21,5279 & 122,1864 \\
$\hat{\beta_{1}}$ & 0,0378 & 0,0810 & 0,0252 & 0,0758 \\
$\hat{\beta_{2}}$ & 1,7634 & 9,0919 & 1,3221 & 10,3333 \\
$r_{y \hat{y}}$ & 0,923 & 0,875 & 0,944 & 0,877 \\
\hline
\end{tabular}

Para a alternativa 2, que utiliza o método de atribuição preliminar de índice de local e o modelo proposto por Payandeh e Wang (1995), a expressão que permitiu gerar as curvas de índice de local, apresentadas na Figura 5, foi:

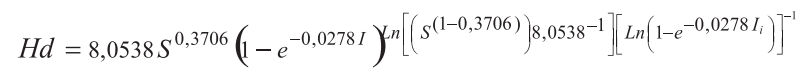

Analisando as Figuras 4 e 5 verificaram-se padrões diferenciados das curvas geradas pelas duas alternativas de classificação da capacidade produtiva. Pela alternativa 2 os dados observados de altura dominante não foram contemplados na Classe de local III, na idade de 22 meses. Na prática, uma classificação da capacidade produtiva utilizando esta alternativa faria com que plantios em idades mais jovens fossem classificados apenas como sites “médios” ou "bons”, e nunca como “ruins”. Embora haja pequena dispersão em altura dominante, em idades inferiores, o emprego da alternativa 2 pode resultar em inconsistências quando se realizar um mapeamento da capacidade produtiva em idades jovens. Dessa forma, para classificação da capacidade produtiva de teca em Tangará da Serra, em idades menores do que 6 anos, o método mais adequado é o da curvaguia (alternativa 1).

R. Árvore, Viçosa-MG, v.32, n.4, p.679-685, 2008 

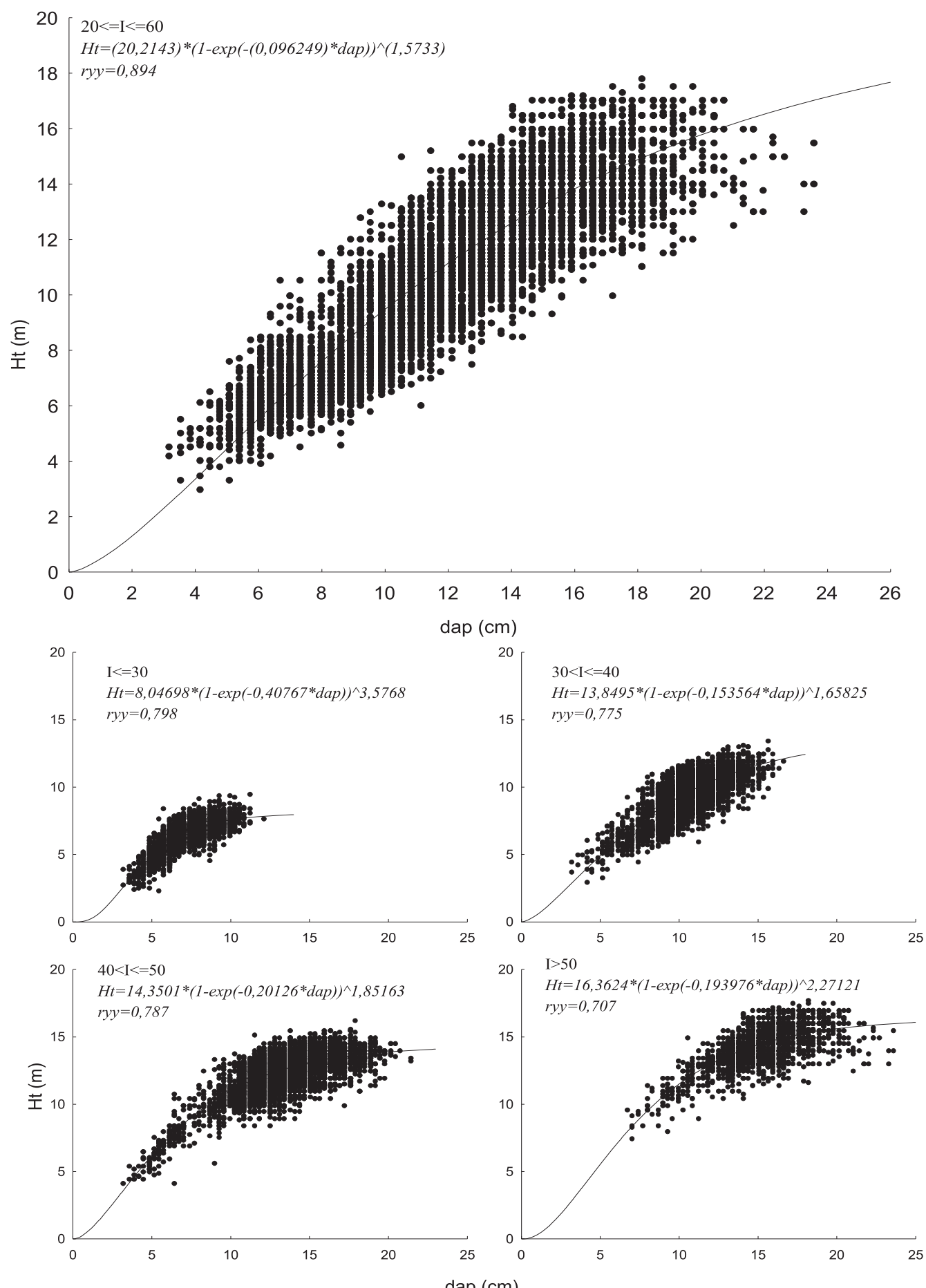

Figura 3 - Relações hipsométricas considerando quatro classes de idade $(I \leq 30 ; 30<I \leq 40 ; 40<I \leq 50 ; I>50)$ e todas idades $(20 \leq I \leq 60)$.

Figure 3 - Hipsometric relations considering four classes of ages $(I \leq 30 ; 30<I \leq 40 ; 40<I \leq 50 ; I>50)$ and all ages $(20 \leq I \leq 60)$.

R. Árvore, Viçosa-MG, v.32, n.4, p.679-685, 2008 


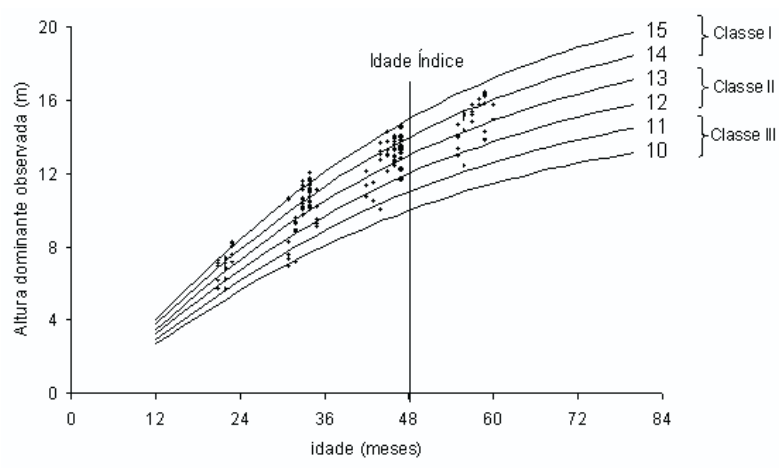

Figura 4 - Curvas de índice de local para teca em Tangará da Serra, geradas pelo método da curva-guia (alternativa 1).

Figure 4-Site index curves for teak in Tangará da Serra, generated by the guide curve method (alternative 1).

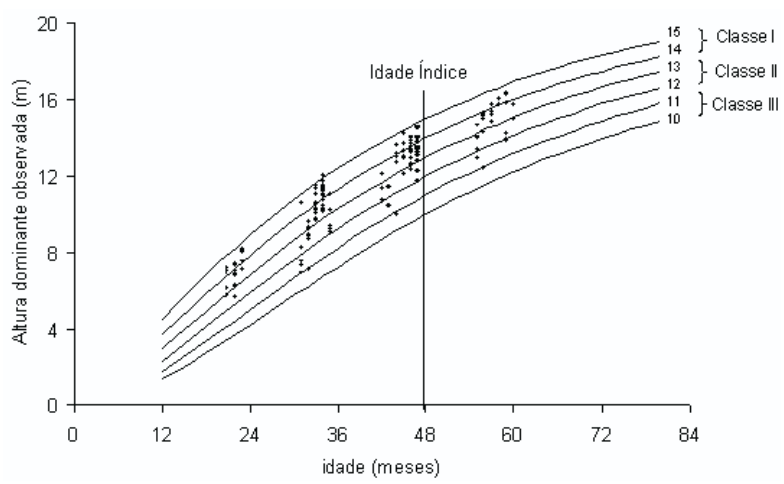

Figura 5 - Curvas de índice de local para teca em Tangará da Serra - MT, geradas pelo método de atribuição preliminar de índices de local (alternativa 2).

Figure 5 - Site index curves for teak in Tangará da Serra, generated by the preliminary attribution of site indexes (alternative 2).

\section{CONCLUSÕES}

Os resultados permitiram concluir que:

- As tendências de crescimento de teca podem ser descritas pelo modelo de Chapman-Richards;

- A alternativa 2 (método de atribuição preliminar de índices de local, utilizando o modelo proposto por Payandeh e Wang, 1995) não é recomendada para classificar a capacidade produtiva de povoamentos eqüiâneos de teca em idades menores que 24 meses;

- Em plantios jovens de teca, com idade inferior a 60 meses, na região de Tangará da Serra - MT, o método da curva-guia para classificação da capacidade produtiva de teca é adequado.

\section{REFERÊNCIAS BIBLIOGRÁFICAS}

CAMPOS, J.C.C.; LEITE, H.G. Mensuração florestal: perguntas e respostas. Viçosa: Editora UFV, 2002. 407p.

HAISHUI, Z. Growth pattern on teak plantations on Hainan island. In: Teak in Asia (edited by $\mathrm{H}$. Wood). FORSPA publication 4, GCP/RAS/I34/ASB. Bangkok. Thailand, p. 119-126, 1993.

HUSCH, B.; MILLER, C.I.; BEERS, T.W. Forest mensuration. 2.ed. New York: Ronald Press, 1972. 409 p.

KEOGH, K. M. Growth rates of teak (Tectona grandis) in the Caribbean/Central-American region. For. Ecol. Manage. n. 35, 1990. p. 311-314. (Short Communication).

MACEDO, R. L. G.; GOMES, J. E.; TSUKAMOTO FILHO, A. A. Análise preliminar do crescimento e fenologia da Tectona grandis L.f. (teca), implantada em parcela de observação na região de Lavras-MG. In: SIMPÓSIO INTERNACIONAL SOBRE ECOSSISTEMAS FLORESTAIS, 5, Curitiba, 1999. Anais... Rio de Janeiro, Biosfera, 1999a. 4p. (CD ROM-BIO 1200).

NOGUEIRA, G.S. Modelagem do crescimento e da produção de povoamentos de Eucalyptus sp. e de Tectona grandis submetidos a desbaste. Viçosa, MG. UFV, 2003. 145f. Tese (Doutorado em Ciências Florestais) Universidade Federal de Viçosa, 2003.

PAYANDEH, B.; WANG, Y. Comparison of the modified Weibull and Richards growth function for developing site index equations. New Forests. n.9. p. 147-155. 1995

PIENAAR, L.V.; TURNBULL, K.J. The ChapmanRichards generalization of Von Bertalanffy's growth model for basal area growth and yield in even-aged stands. Forest Science, v. 19, n. 1, p.2-22, 1973.

REED, D.D.; JONES, E.A.; TOMÉ, M.; ARAÚJO, M.C. Models of potential height and diameter for Eucalyptus globulus in Portugal. For. Ecol. Manage. n. 172, 2003. p. 191-198.

SCHUMACHER, F.X.; HALL, F.S. Logarithmic expression of the timber volume. Journal of Agriculture Research, Lahore, v.47, n.9, p.719-34, 1933.

ZEIDE, B. Analysis of growth equations. Forest Science, v. 39, n. 3, p. 594-616. 1993.

R. Árvore, Viçosa-MG, v.32, n.4, p.679-685, 2008 